\title{
Clinical Study \\ Refractory Status Epilepticus: Experience in a Neurological Intensive Care Unit
}

\author{
O. H. Hernandez, ${ }^{1}$ J. F. Zapata, ${ }^{2}$ M. Jimenez, ${ }^{2}$ M. Massaro, ${ }^{3}$ A. Guerra, ${ }^{1}$ J. C. Arango, ${ }^{1}$ \\ J. D. Ciro, ${ }^{1}$ H. Delgado, ${ }^{1}$ and J. I. Suarez ${ }^{4}$ \\ ${ }^{1}$ Instituto Neurologico de Colombia, Neurological Intensive Care Unit, Calle 55 No. 46-36, 050012 Medellín, Colombia \\ ${ }^{2}$ Instituto Neurologico de Colombia, Clinical Neurophysiology Unit, Medellin, Colombia \\ ${ }^{3}$ Instituto Neurologico de Colombia, Research and Education Department, Medellin, Colombia \\ ${ }^{4}$ Baylor College of Medicine Vascular Neurology and Neurocritical Care Division, Department of Neurology, Houston, TX, USA
}

Correspondence should be addressed to O. H. Hernandez; olgahdezo@gmail.com

Received 2 May 2013; Revised 30 July 2013; Accepted 12 August 2013; Published 2 January 2014

Academic Editor: Marieke Van Zoelen

Copyright (C) 2014 O. H. Hernandez et al. This is an open access article distributed under the Creative Commons Attribution License, which permits unrestricted use, distribution, and reproduction in any medium, provided the original work is properly cited.

\begin{abstract}
Introduction. Refractory status epilepticus (RSE) has significant morbidity and mortality, and its management requires an accurate diagnosis and aggressive treatment. Objectives. To describe the experience of management of RSE in a neurological intensive care unit (NeuroICU) and determine predictors of short-term clinical outcome. Methods. We reviewed cases of RSE from September 2007 to December 2008. Management was titrated to findings on continuous video EEG (cVEEG). We collected patients' demographics, RSE etiology, characteristics of seizures, cVEEG findings, treatments, and short-term outcome. Control of RSE was to achieve burst suppression pattern or electrographic cessation of ictal activity. Results. We included 80 patients; $63.8 \%$ were in coma, $25 \%$ had subclinical seizures, and $11.3 \%$ had focal activity. $51.3 \%$ were male and mean age was 45 years. Etiology was neurological lesion in $75.1 \%$, uncontrolled epilepsy in $20 \%$, and systemic derangements in $4.9 \%$. $78.8 \%$ were treated with general anesthesia and concomitant anticonvulsant drugs. The control of RSE was $87.5 \%$ of patients. In-hospital mortality was $22.5 \%$. The factors associated with unfavorable short-term outcome were coma and age over 60 years. Conclusions. RSE management guided by cVEEG is associated with a good seizure control. A multidisciplinary approach can help achieve a better short-term functional outcome in noncomatose patients.
\end{abstract}

\section{Introduction}

Status epilepticus (SE) is a medical emergency, due to significant morbidity and mortality and requires prompt attention and adequate management. In different series, the SE mortality varies between 17 and 26\%, and approximately 10 to $23 \%$ of the patients that survived presented some degree of neurological impairment [1-4]. In the Intensive Care Unit (ICU) there are two groups of patients with SE: patients with multiple episodes of clinical seizures who have various response to initial management, and there are individuals admitted for other reasons who developed subclinical ictal episodes during their stay in the ICU. The latter is classified as nonconvulsive status epilepticus (NCSE) $[1,4,5]$.
It is estimated that NCSE represents between 25 and 50\% of all SE cases, but in the critically ill patient this entity could have a greater incidence. Various publications report that $10 \%$ of comatose patients suffer from NCSE, being the incidence much higher in the neurocritical care unit (NeuroICU), where about $34 \%$ of patients can have altered consciousness [6-11].

Continuous video electroencephalogram (cVEEG) monitoring is a necessary tool in patients with SE to establish adequate diagnosis, classification of disease, treatment guidance, and followup until ictal activity suppression is reached [12$14]$.

It is calculated that 10 to $40 \%$ of all patients with SE evolve to a refractory status epilepticus (RSE). In general, 
this progression is time dependent with early management being advocated to achieve control $[15,16]$. RSE is defined as either the absence of response to a first-line antiepileptic drug (benzodiazepines) and to one or two second-line drugs (phenytoin, phenobarbital, or valproate) or persistent ictal activity despite one- or two-hour treatment. RSE has a high mortality (up to 50\%) and usually requires suppression of ictal electrographic activity $[1,15,16]$.

The RSE approach in the NeuroICU needs a multidisciplinary team with the participation of neurophysiologists, intensive care specialists, neurologists, and in some cases, neurosurgeons. Control of RSE demands the use of multiple anticonvulsant drugs (AEDs), high-dose sedatives, and vasopressive support. Since these medications typically induce respiratory depression and hemodynamic instability, mechanical ventilatory support and invasive monitoring are necessary $[1,15-22]$.

RSE management is based on case reports and experience from large tertiary-care centers. Most investigators recommend two types of management: (1) administration of sedatives to achieve elimination of electrographic ictal activity and reaching pharmacological burst suppression pattern and (2) a less aggressive alternative, without sedation, using AEDs in high doses, in a step-up approach, guided by the improvement observed by cVEEG monitoring [23-30].

We set out to investigate several aims: to describe the experience of approach and management of RSE in a NeuroICU; to compare outcomes according to the type of RSE (comatose and noncomatose); and to determine the predictors of short-term functional prognosis.

\section{Patients and Methods}

We conducted a retrospective study that included all patients in the NeuroICU at the Instituto Neurologico de Colombia (Medellin, Colombia), in whom the diagnosis of RSE was made between September 2007 and December 2008.

In all patients, pharmacologic treatment was guided by cVEEG monitoring. A Cadwell equipment with 32 channels and surface electrodes was used, following the protocol according to the International 10-20 System; also, electrodes were installed for electrocardiogram monitoring, surface electromyography in both deltoids, and in some cases, electrooculogram, depending on the clinical symptoms reported. The cVEEG interpretation was made by two trained neurophysiologists who sent a verbal and written report every twelve hours. Analysis was performed using Easy III (Cadwell Inc., Kennewick, WA) software, evaluating the recording, at least in three montages, modifying sensitivity and speed when necessary for the analysis.

Electrographic criteria defined by Young et al. and modified by Chong and Hirsch for diagnosing NCSE were used $[31,32]$. The status epilepticus classification was performed according to clinical features: coma (ICU patient without waking who develops EER), subtle status (patient with acute electrographic seizure after initial treatment of status epilepticus), and refractory partial status (patient with electroclinical findings of refractory focal seizures).
Our group defined RSE as SE refractory to first-line (benzodiazepines) and second-line (phenytoin, phenobarbital, or valproate) AEDs. A staff conformed by NeuroICU doctors and clinical neurophysiologists defined RSE classification and management of patients according to institutional algorithm. Our therapeutic approach included general anesthesia (continuous infusion of midazolam, propofol, and/or thiopental) and concomitant AEDs (valproate, levetiracetam, topiramate, and phenobarbital, among others). For therapeutic purposes, patients' age, hemodynamic status, comorbidities, and level of consciousness as determined by the GCS [33], were considered. Patients' management algorithm is presented in Figure 1.

In those patients receiving deep sedation, the management goal was achievement of a 50/50 burst suppression pattern on EEG pattern (50\% burst and 50\% suppression) for at least 24 hours. Subsequently, our management protocol called for a $5 \%$ /hour dose tapering of the medication used. In those patients receiving management with only stepup AEDs, the therapeuticgoal was accomplishment of EEG control of ictal activity. In those patients with successful control of RSE, we performed cVEEG monitoring every 48 hours or before that if the patients' condition required it for evaluation of relapse of ictal activity, which we called breakthrough seizures.

We collected the following data from the medical records: patients' demographics, etiology of RSE, the Acute Physiology And Chronic Health Evaluation (APACHE) II score, Status Epilepticus Severity Score (STESS), clinical characteristics of seizures, EEG findings, treatments received, and shortterm outcome. The latter was defined as in-patient mortality and ability to regain independence in activities of daily living at one month as defined by a modified Rankin scale (mRs) score $\leq 2$ [34]. The APACHE II score was selected because it is a good validated indicator of underlying disease severity [35]. APACHE II score ranges from 0 to 71 , and higher values indicate more severe disease state. The STESS has been suggested as a good indicator of severity of SE [36]. STESS ranges from 0 to 6 , and higher values indicate more severe derangement. Generally, a nonaggressive management was used in patients with low scores, and in patients with high scores (more than 2 points), deep sedation was performed.

2.1. Statistical Analysis. We used SPSS version 15.0 for processing and analysis of our data (Chicago, IL, 2006). All data were analyzed for normality, and appropriate statistical modeling was used accordingly. A univariate analysis was performed with descriptive statistics: proportion comparison for categorical variables with $\chi^{2}$ association test or Fisher exact test and mean differences for quantitative variables with student's $t$-test or Mann-Whitney test. For statistical significance, a two-tailed $P$ value $<0.05$ was considered significant.

The data are presented according to the type of RSE (coma, subtle, and partial), treatment type (general anesthesia and AED), and according to the gold of treatment (control and no control of RSE). Additionally, the cohort of patients 


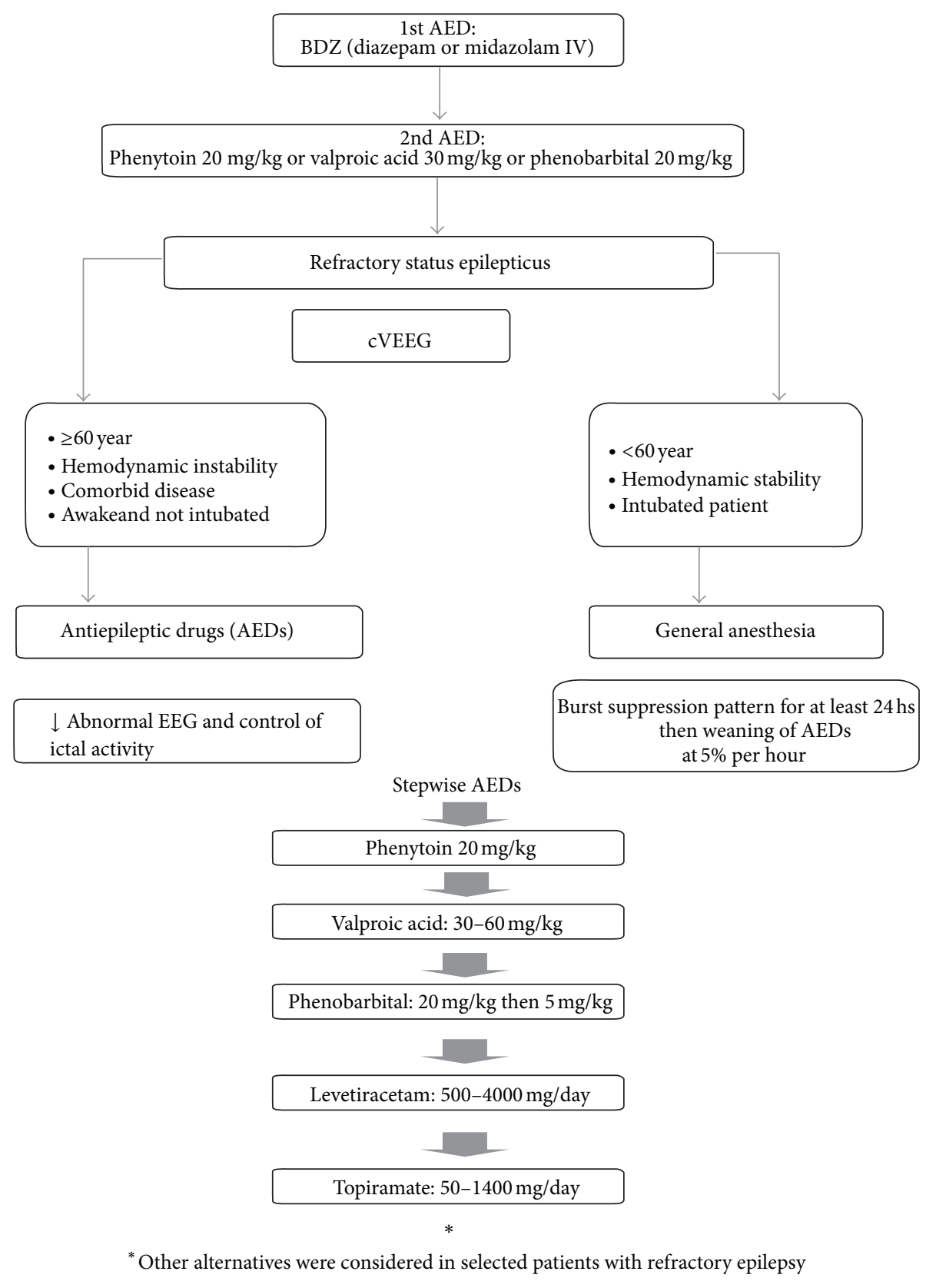

FIGURE 1: Treatment algorithm for RSE: guidelines from the Instituto Neurologico de Colombia.

was recategorized according to the presence or absence of coma (subtle and partial refractory state).

We then performed a multivariate analysis using a forward stepwise logistic regression (Forward Stepwise [RV] Method). The dependent variable was functional prognosis as determined by mRs one month after diagnosis of RSE dichotomized to good functional outcome (mRs $\leq 2$ ) and poor functional outcome $(\mathrm{mRs}>2)$. We included in the multivariate model those factors with $P$ values $<0.25$. Finally we included those independent variables with relevant clinical significance that explained the functional prognosis. The results of our multivariate model are presented as odds ratio (OR) with their respective confidence interval (95\% CI) for variables with $P$ values $<0.05$. In addition, we included interaction terms and investigated collinearity among the covariates of interest.

\section{Results}

During a 15-month period, 619 patients were admitted to our NeuroICU. Of those 80 (12.9\%) had RSE. Most patients were men $(51.3 \%)$ with a mean age of 45 years. The most frequent clinical findings in the patients who developed RSE were altered level of consciousness (77.5\%) and minor motor findings (rhythmical twitching of facial muscles, ocular muscles, or distal extremities) (30\%). The most common etiology 
TABLE 1: Demographic characteristics of patients.

\begin{tabular}{lc}
\hline Demographic characteristics & $n=80$ \\
\hline Age (years) & $45(19.5)$ \\
Sex (male) & $41(51.3)$ \\
GCS on admission to the NeuroICU & $10(8-14)$ \\
APACHE II Score & $15(9-20)$ \\
STESS & $4(2-4)$ \\
Days since seizure started & $3(1.5-7)$ \\
Etiology of RSE & \\
Acute neurological lesion & $60(75.1)$ \\
$\quad$ Stroke & $29(48.3)$ \\
$\quad$ Hemorrhagic & $17(58.6)$ \\
$\quad$ Ischemic & $12(41.4)$ \\
CNS infection & $9(15)$ \\
TBI & $8(13.4)$ \\
Anoxic encephalopathy & $7(11.7)$ \\
CNS tumors & $5(8.3)$ \\
Other (intracranial hypertension syndrome, & $2(3.3)$ \\
neuroleptic malignant syndrome) & $16(20)$ \\
Preexisting epilepsy flare & $4(4.9)$ \\
Systemic acute lesion &
\end{tabular}

Data are number (\%), mean (SD), or median (IQR).

GCS: Glasgow Coma Scale; NeuroICU: neurological intensive care unit; APACHE II: The Acute Physiology and Chronic Health Evaluation Score; STESS: Status Epilepticus Severity Score; RSE: refractory status epilepticus; CNS: central nervous system; TBI: traumatic brain injury.

of RSE was acute neurological lesion in $75.1 \%$ of the patients. The demographic characteristics of our cohort are shown in Table 1.

All the patients included in this study met NCSE criteria as defined by cVEEG monitoring and the medical staff caring for them.

The characteristics of the patients who developed RSE were different in the three study groups: RSE coma patient, subtle status, and refractory partial status. Most of these patients were comatose $(63.8 \%)$, and most (94.1\%) were admitted with acute neurologic injury, and its evolution developed an RSE. The second RSE group (subtle status) accounted for $25 \%$, of which the majority (75\%) had a history of epilepsy and after initial management of status epilepticus persisted with electrographic seizures. In the latter group, $11.3 \%$ of patients had partial subclinical RSE. Comatose RSE patients had the worst condition with the highest scores according to baseline characteristics (Table 2).

We found that the entire cohort of patients received benzodiazepines as first-line medication: 75\% midazolam, $15 \%$ diazepam, and 10\% lorazepam. In addition, we found that the second-line medications were phenytoin $(76.3 \%)$, valproate $(22.5 \%)$, and phenobarbital $(1.2 \%)$.

We instituted profound sedation in $78.8 \%$ of patients with RSE and step-up AEDs administration in all of them. We found that for coma induction, the most frequently used medication was midazolam $(87.3 \%)$, and levetiracetam and topiramate were the most frequently administered newergeneration AEDs for continuing the step-up management of RSE (97.5\% and $77.5 \%$, resp.) (Table 3 ). The most frequent complication associated with profound sedation use was hypotension, without significant differences between the medications used (midazolam 34.5\%, propofol $32.1 \%$, and thiopental 33.3\%). One patient experienced propofolinfusion syndrome.

Those patients managed with general anesthesia required longer treatment and more medications compared with patients managed only with AEDs (Table 4). In comatose RSE, general anesthesia management was used in $90.2 \%$ of patients; the remaining comatose patients (9.8\%) were treated only with AED because their base condition did not allow any aggressive treatment; of those, 2 died (40\%) and additionally, a patient did not reach control (20\%). When compared with the aggressive management subgroup, the mortality was $30.4 \%$, and there was no seizure control in $10.9 \%$.

The rate of general anesthesia management in subtle form was $85 \%$, and a nonaggressive management was performed in the remaining $(15 \%)$ because of their initial condition (nonintubated) and also a STESS less than 2. Whereas all patients with partial RSE received step-up AED only.

The median duration of cVEEG monitoring was 120 hours (IQR: 120-132). The EEG patterns during treatment induction were as follows: localized interictal epileptiform activity in $80 \%$; periodic/pseudoperiodic generalized discharges [GPED] in 95\%, and periodic/pseudoperiodic lateralized discharges [PLEDs or BiPLEDs] in 40\%. According to electrographic classification of comatose RSE, $58.8 \%$ of the patients had a GED-coma pattern (Generalized Epileptiform Discharges).

RSE was successfully controlled within 3 days in $87.5 \%$ of patients, with a longer median time for those patients treated with general anesthesia compared to those who received AEDs only (Table 4). Only two patients of those who achieved successful control of RSE control presented breakthrough seizures $(2.9 \%)$. The RSE control was achieved in $88.2 \%$ of patients in coma and $86.6 \%$ of the non-comatose group; $66 \%$ and $50 \%$ of patients that did not reached control of RSE died in the coma and non-coma group, respectively. The mortality in uncontrolled patients was significantly higher compared to those who achieved control of RSE (60\% and $17.1 \%$, resp., $P=$ 0.007). Similarly, the no control is associated with a shortterm unfavorable outcome in univariate analysis (Table 5).

In univariate analysis, we found that the only factor associated with lack of control of RSE was stroke (Table 6). In addition, our data show that patients with RSE who are comatose required longer treatment days and had higher mortality and poorer clinical outcome, in contrast to those who were non-comatose (Table 7).

Overall in-hospital mortality was $22.5 \%$. All patients that died did so during their stay in the ICU, of which $88.9 \%$ belonged to the comatose group and the $11.1 \%$ to the noncomatose group. Mortality in the comatose group resulted from their initial neurological injury; from the noncomatose group, two patients died who were part of the subgroup of subtle state: a patient with endocarditis and 
TABLE 2: Patient's characteristics according to RSE type.

\begin{tabular}{|c|c|c|c|c|}
\hline & $\begin{array}{l}\text { Coma } \\
n=51\end{array}$ & $\begin{array}{l}\text { Subtle } \\
n=20\end{array}$ & $\begin{array}{c}\text { Partial } \\
n=9\end{array}$ & $P$ value \\
\hline Age & $48(18.8)$ & $33(17.4)$ & $53(18.2)$ & 0.004 \\
\hline GCS at NeuroICU admission & $9(7-13)$ & $10(8-14)$ & $14(11-14)$ & 0.005 \\
\hline APACHE II Score & $17(12-21)$ & $14(7-16)$ & $8(3-9)$ & $<0.0001$ \\
\hline STESS & $4(4-4)$ & $2(2-2)$ & $1(0-2)$ & $<0.0001$ \\
\hline Time until treatment in NeuroICU, days & $1(0.5-7)$ & $2(1-6)$ & $1(1-5.5)$ & 0.92 \\
\hline \multicolumn{5}{|l|}{ Etiology } \\
\hline Acute neurological lesion & $48(94.1)$ & $5(25)$ & $7(77.8)$ & $<0.0001$ \\
\hline Acute systemic lesion & $3(5.9)$ & 0 & $1(11.1)$ & 0.39 \\
\hline Epilepsy & 0 & $15(75)$ & $1(11.1)$ & $<0.0001$ \\
\hline \multicolumn{5}{|l|}{ Treatment type } \\
\hline Deep sedation & $46(90.2)$ & $17(85)$ & 0 & $<0.0001$ \\
\hline Only AED & $5(9.8)$ & $3(15)$ & $9(100)$ & $<0.0001$ \\
\hline
\end{tabular}

Data are number (\%), mean (SD), or median (IQR).

GCS: Glasgow Coma Scale; APACHE II: The Acute Physiology and Chronic Health Evaluation Score; STESS: Status Epilepticus Severity Score; NeuroICU: neurological intensive care unit; AED: antiepileptic drugs.

TABLE 3: AEDs administered for management of RSE.

\begin{tabular}{lcc}
\hline Drug & $\begin{array}{c}n=80 \\
\text { No. }(\%)\end{array}$ & $\begin{array}{c}\text { Dose used } \\
\text { Lowest-highest }\end{array}$ \\
\hline $\begin{array}{l}\text { Coma induction } \\
\text { Midazolam }\end{array}$ & $55(87.3)$ & $0.0006-0.1^{\ddagger}$ \\
Propofol & $28(44.4)$ & $0.2-12^{\S}$ \\
Thiopental & $3(4.8)$ & $1-4.5^{\S}$ \\
AEDs & & \\
Levetiracetam & $78(97.5)$ & $1000-4000^{\S}$ \\
Phenytoin & $67(83.8)$ & $250-500^{\S}$ \\
Topiramate & $63(78.8)$ & $50-1400^{\S}$ \\
Valproate & $62(77.5)$ & $450-3500^{\S}$ \\
Phenobarbital & $39(48.8)$ & $150-500^{\S}$ \\
Other* & $54(67.5)$ & \\
\hline
\end{tabular}

${ }^{*}$ Carbamazepine, lamotrigine, pregabalin, vigabatrin, and gabapentin.

${ }^{\ddagger} \mathrm{mg} / \mathrm{kg} /$ hour.

${ }^{9} \mathrm{mg} / \mathrm{kg}$.

${ }^{\$} \mathrm{mg} /$ day.

AED: antiepileptic drugs; RSE: refractory nonconvulsive status epilepticus.

embolic stroke with APACHE 23 and the other one with herpes encephalitis who died with propofol syndrome.

At the first month followup, $48.4 \%$ of patients had a good functional outcome. The proportion of patients with $\mathrm{mRs}$ $\leq 2$ at the same period followup was significantly higher in the non-comatose group, compared to comatose RSE group (66.7\% for partial refractory status and 65\% for subtle type versus $21.6 \%$ in coma RSE, $P<0.0001$ ) (Figure 2 ).

In univariate analysis the factors associated with unfavorable functional outcome included the following: age, stroke, acute neurological lesion, coma, and uncontrolled RSE; whereas underlying epilepsy was associated with good outcome (Table 5).
TABle 4: Patient's characteristics according to treatment type.

\begin{tabular}{lccc}
\hline Characteristics & $\begin{array}{c}\text { General } \\
\text { anesthesia } \\
n=63\end{array}$ & $\begin{array}{c}\text { AEDs only } \\
n=17\end{array}$ & $P$ value \\
\hline Age & $43(19.5)$ & $52(18.9)$ & 0.1 \\
GCS at NeuroICU & $9(7-13)$ & $13(10-14)$ & 0.003 \\
admission & $16(11-21)$ & $8(4-13)$ & 0.001 \\
APACHE II Score & $49(77.8)$ & $7(41.2)$ & 0.003 \\
STESS 3-6 & $5(5-8)$ & $4(3-5)$ & 0.001 \\
RSE treatment days & $3.2(0.8)$ & $2.5(0.7)$ & 0.003 \\
Average AED used &
\end{tabular}

Data are number (\%), mean (SD), or median (IQR).

GCS: Glasgow Coma Scale; APACHE II: The Acute Physiology and Chronic Health Evaluation Score; STESS: Status Epilepticus Severity Score; RSE: refractory status epilepticus; AED: antiepileptic drugs.

In multivariate analysis, coma and age $\geq 60$ years were the only factors independently associated with unfavorable short-term functional outcome (Table 8).

\section{Discussion}

RSE is an underdiagnosed entity, but, with cVEEG monitoring in the NeuroICU, the reports of this disease are increasing $[1,6,7]$. In our center, the incidence of RSE in a 15-month follow-up period was $12.9 \%$.

Patients experiencing RSE can become comatose or have other neurological manifestations such as blinking, sucking, or subtle motor movements in hands, face, or feet $[5,7-11]$. The latter group would potentially benefit from cVEEG. In a recent questionnaire directed to neurologists concerning cVEEG use in the management of NCSE, the following factors were considered as the principal indications for its use: previous history of seizures (89\%), abnormal eye movements (85\%), and altered awareness (69\%) [13]. In our cohort, 
TABLE 5: Univariate analysis of factors associated with unfavorable short-term functional outcome.

\begin{tabular}{lccc}
\hline Factor & OR & $95 \%$ CI & $P$ value \\
\hline Age $>60$ years & 6 & $1.6-22.46$ & 0.04 \\
APACHE II Score $\geq 15$ & 2.81 & $1.10-7.19$ & 0.028 \\
Etiology: stroke & 3.4 & $1.18-9.77$ & 0.019 \\
Etiology: CNS infection & 2.27 & $0.44-11.77$ & 0.26 \\
Etiology: anoxia/hypoxia & 1.55 & $0.28-8.56$ & 0.47 \\
Etiology: brain tumor & 0.37 & $0.05-2.38$ & 0.27 \\
Etiology: traumatic brain injury & 1.9 & $0.36-10.13$ & 0.36 \\
Systemic abnormalities & 1.85 & $0.18-18.64$ & 0.51 \\
Underlying epileptic disease & 0.13 & $0.03-0.45$ & 0.001 \\
Acute neurological lesion & 4.69 & $1.6-13.78$ & 0.003 \\
Coma & 6.9 & $2.5-19.07$ & $<0.0001$ \\
Previous treatment time $>10$ hours & 0.63 & $0.19-2.01$ & 0.43 \\
Uncontrolled RSE & 15.8 & $1.49-280.5$ & 0.03 \\
\hline
\end{tabular}

OR: Odds ratio; CI: confidence interval; APACHE II: The Acute Physiology and Chronic Health Evaluation Score; CNS: central nervous system; RSE: refractory status epilepticus.

TABLE 6: Univariate analysis of factors associated with lack of control of RSE.

\begin{tabular}{lccc}
\hline \multirow{2}{*}{ Factor } & \multicolumn{3}{c}{ Uncontrolled RSE $n=10$} \\
& OR & $95 \%$ CI & $P$ value \\
\hline Age $>60$ years & 0.58 & $0.11-2.98$ & 0.4 \\
APACHE II Score $>15$ & 1.41 & $0.36-5.46$ & 0.43 \\
Etiology: stroke & 5.09 & $1.2-21.56$ & 0.023 \\
Etiology: neuroinfection & 4.57 & $0.93-22.42$ & 0.08 \\
Etiology: anoxia/hypoxia & 0.4 & $0.03-6.91$ & 0.65 \\
Etiology: brain tumour & 1.00 & $0.14-7.19$ & 0.57 \\
Etiology: traumatic brain injury & 0.63 & $0.07-5.53$ & 0.94 \\
Systemic abnormalities & 1.21 & $0.12-11.43$ & 0.66 \\
Underlying epileptic disease & 0.29 & $0.03-2.44$ & 0.41 \\
Acute neurological lesion & 0.83 & $0.74-0.98$ & 0.11 \\
Coma & 0.83 & $0.21-3.23$ & 0.52 \\
General anesthesia & 0.91 & $0.17-4.77$ & 0.64 \\
Previous treatment time over 10 hours & 1.09 & $0.2-5.68$ & 0.64 \\
\hline
\end{tabular}

APACHE II: The Acute Physiology and Chronic Health Evaluation Score; OR: Odds ratio; $\mathrm{CI}$ : confidence interval.

the most common indications for cVEEG were altered awareness $(77.5 \%)$, and subtle motor movements (30\%).

Around $33-50 \%$ of SE cases occur in patients without past medical history of epilepsy and in the context of an acute condition involving the central nervous system, like stroke (20-36\%), head trauma (1-26\%), metabolic alterations or drug withdrawal (7-26\%), central nervous system infections $(3-14 \%)$, or tumors $(5-24 \%)[1,2,4]$. In our group, the principal cause of SE was acute neurological lesion (75.1\%), with a higher percentage compared to those reported in the literature, with stroke and central nervous system infection being the principal causes. This finding could be explained by the fact that our patients were treated in a NeuroICU.

Our institution is a tertiary referral neurological center, and often times patients' transfer and admission to the

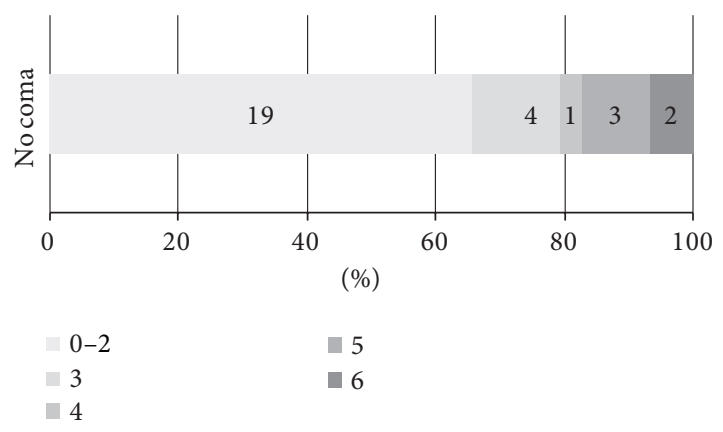

(a)

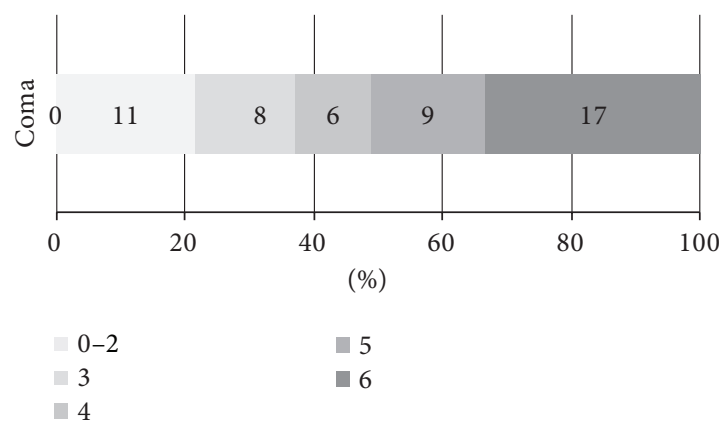

(b)

Figure 2: Modified Rankin Scale score in comatose and noncomatose patients.

NeuroICU with generalized and partial SE are delayed. Therefore, patients with SE who were admitted to our NeuroICU had already received some treatment and met criteria for subclinical or partial NCSE. In fact, most of our patients with RSE were comatose (63.8\%), due to the fact that they had experienced severe neurological lesions and greater susceptibility to ictal events $[10,15,16]$.

For adequate control of RSE, as we described earlier, the literature suggests two management alternatives: coma induction reaching a burst suppression pattern in the cVEEG and a less aggressive treatment with high-dose AED. Whether one treatment should be chosen over the other has been a subject of debate [15-22]. In recent studies, the STESS showed the power to stratify patients according to SE severity, allowing practitioners to choose the best treatment strategy for each patient [36]. We employed STESS in our cohort, which was one of the tools used to define the treatment. Overall, in patients with low scores, a nonaggressive management was introduced; and in patients with high scores (more than 2 points), deep sedation was used.

For coma induction, medications like barbiturates, propofol, or midazolam are used. A meta-analysis evaluating these three medications in the management of RSE suggested that although pentobarbital seems to be superior to propofol and midazolam in regards to effectiveness of RSE control (to stop seizures and to avoid their relapse), this drug is associated with more hypotensive episodes, demanding vasopressor use [15]. Considering this evidence and due to comparable mortality between these medications, our group employs more frequently midazolam followed by propofol, 
TABLE 7: Outcome of RSE according to level of consciousness.

\begin{tabular}{lccc}
\hline Outcome & Coma & Noncoma & $n=29$ \\
& $n=51$ & $5(3.5-6)$ & $P$ value \\
\hline Treatment days & $5(4-8)$ & $8(4.7-13)$ & 0.057 \\
Mechanical ventilation days & $12(8-18)$ & $8(27.6)$ & 0.03 \\
Tracheostomy & $40(78.4)$ & $3(10.3)$ & $<0.0001$ \\
Gastrostomy & $15(29.4)$ & $13(44.8)$ & 0.05 \\
Infectious complications (VAP, UTI, BSI) & $21(41.2)$ & $9(31)$ & 0.75 \\
Vasopressor use & $24(47.1)$ & $8(3-12.5)$ & 0.16 \\
NeuroICU days & $14(10-22)$ & $25(86.2)$ & $2(6.9)$ \\
RSE Control & $45(88.2)$ & $10(34.5)$ & 0.0001 \\
In-hospital mortality & $16(31.4)$ & 0.012 \\
Unfavorable mRs at 1 month & $40(78.8)$ & $<0.0001$ \\
\hline
\end{tabular}

Data are number (\%), mean (SD), or median (IQR).

VAP: ventilator associated pneumonia, UTI: urinary tract infection; BSI: blood stream infection; NeuroICU: neurological intensive care unit; RSE: refractory status epilepticus; mRs: modified Rankin scale.

TABLE 8: Prognostic factors for unfavorable short-term functional outcome in multivariate analysis.

\begin{tabular}{lcccc}
\hline & Factor & OR & $95 \%$ CI & $P$ value \\
\hline \multirow{2}{*}{ Nonfavorable $m$ Rs $(\geq 3)$} & Coma & 8.84 & $2.62-29.84$ & $<0.0001$ \\
& Age $>60$ years & 6.36 & $1.44-28$ & 0.014 \\
\hline
\end{tabular}

leaving barbiturates only in patients with head trauma associated with refractory high intracranial pressure, where the barbiturate could be used for both purposes.

According to the literature, coma-inducing medications titrated to attain EEG burst suppression pattern were associated with a significant lower incidence of breakthrough seizures [15]. This suggests that the burst suppression pattern associated with pharmacological sedation is produced by the effect over the GABA receptors of the cortical-thalamus pathways, generating a cortical synchronization that could explain the control of ictal activity. This argument explains our reason to induce burst suppression pattern in most of our patients (78.8\%): $90 \%$ comatose RSE and $85 \%$ subclinical RSE.

In the last few years, several reports have been published concerning the use of new AEDs for control of RSE, such as levetiracetam and topiramate, which have demonstrated favorable results in these patients [37-43]. Our management algorithm also included the use of these drugs. In fact, levetiracetam and topiramate were the most-commonly used new-generation AED (97.5\% and 77.5\%) in our patients.

The management of patients with RSE requires a multidisciplinary team, and continuous hemodynamic monitoring is needed to minimize possible complications associated to the medical management. In our patients, hypotension was the most common medical complication (about 33\% of the cases) which is similar to the published literature $[15,17,21$, 22].

The other important component of the neuromonitoring of patients with RSE is cVEEG which is noninvasive and enables assessment of cerebral function and multimodal monitoring in the neuroICU [12-14]. The literature suggests that $4-48 \%$ of the comatose critically ill patients may have nonconvulsiveictal activity. Therefore, cVEEG monitoring is indispensable for detection and management of these episodes [9-11]. cVEEG monitoring has been useful in the classification of comatose RSE, based upon EEG patterns. Lateralized Epileptiform Discharge (LED) coma is the result of focal lesions leading to focal or lateralized discharges; conversely, Generalized Epileptiform Discharge (GED) coma is the consequence of diffuse central nervous system discharges due to infections, intoxications, or occupying space lesions that produce intracranial hypertension, like tumors [11]. In our cohort, $58.8 \%$ of patients had a GED-coma RSE pattern.

It is important to emphasize that despite having new AEDs and cVEEG, RSE progression is generally time dependent, and it is imperative to avoid delays in its management. In addition, new evidence has shown that it is possible to achieve functional and cognitive recovery after prolonged SE $[44,45]$. In this cohort RSE was successfully controlled within 3 days in $87.5 \%$ of patients; this is consistent with data reported in different series, and possibly the time to achieve control is determined by the underlying etiology of severe neurological injury.

Our review of the literature showed that mortality from RSE can reach 50\%; in this study, in-hospital mortality was $22.5 \%$ at one-month followup; about half of the patients regained their independent daily activities, with a higher proportion in the non-comatose group. This fact supports recent findings about aggressive management of these patients to obtain an acceptable recovery $[44,45]$.

Overall, RSE is associated with high morbidity and mortality, and the reported risk factors of poor prognosis are the following: elderly, mechanical ventilation necessity, coma, hypoxic ischemic encephalopathy lesions, and associated comorbidities [15-18]. Our multivariate analysis showed that 
coma and age over 60 years were the only predictors independently associated with unfavorable short-term functional outcome. These may indicate that these variables should be considered in future studies for the management of patients with RSE $[44,45]$.

Our study has several limitations. Our analysis was retrospective and as such is subject to bias. In addition, the reported cohort stems from a single center, and the results may not be applicable to all patients with RSE. Furthermore, our sample size is relatively small and our results may have to be replicated prospectively and in a larger sample population. Lastly, we do not have data on long-term clinical outcomes including quality of life. The latter should be included in future studies.

In summary, our study includes patients with RSE managed by a multidisciplinary team following a defined management protocol. Our data suggest that patients with RSE can improve, even though comatose patients may have a grimmer prognosis. More publications and randomizedcontrolled trials are required to clarify the best management and monitoring for patients with RSE.

\section{Acknowledgments}

The authors wish to thank Dr. Londoño LV and Dr. C. S. Uribe for their initial contribution to this study.

\section{References}

[1] K. J. Abou Khaled and L. J. Hirsch, "Updates in the management of seizures and status epilepticus in critically ill patients," Neurologic Clinics, vol. 26, no. 2, pp. 385-408, 2008.

[2] H. Arif and L. J. Hirsch, “Treatment of status epilepticus," Seminars in Neurology, vol. 28, no. 3, pp. 342-354, 2008.

[3] J. W. Y. Chen and C. G. Wasterlain, "Status epilepticus: pathophysiology and management in adults," The Lancet Neurology, vol. 5, no. 3, pp. 246-256, 2006.

[4] M. A. Mirski and P. N. Varelas, "Seizures and status epilepticus in the critically ill," Critical Care Clinics, vol. 24, no. 1, pp. 115$147,2008$.

[5] R. J. DeLorenzo, E. J. Waterhouse, A. R. Towne et al., "Persistent nonconvulsive status epilepticus after the control of convulsive status epilepticus," Epilepsia, vol. 39, no. 8, pp. 833-840, 1998.

[6] A. R. Towne, E. J. Waterhouse, J. G. Boggs et al., "Prevalence of nonconvulsive status epilepticus in comatose patients," Neurology, vol. 54, no. 2, pp. 340-345, 2000.

[7] J. Claassen, S. A. Mayer, R. G. Kowalski, R. G. Emerson, and L. J. Hirsch, "Detection of electrographic seizures with continuous EEG monitoring in critically ill patients," Neurology, vol. 62, no. 10, pp. 1743-1748, 2004.

[8] K. G. Jordan, "Continuous EEG and evoked potential monitoring in the neuroscience intensive care unit," Journal of Clinical Neurophysiology, vol. 10, no. 4, pp. 445-475, 1993.

[9] R. Maganti, P. Gerber, C. Drees, and S. Chung, "Nonconvulsive status epilepticus," Epilepsy and Behavior, vol. 12, no. 4, pp. 572586, 2008.

[10] J. L. Fernández-Torre, "Estado epiléptico no convulsivo en adultos en coma," Revue Neurologique, vol. 50, no. 5, pp. 300-308, 2010.
[11] G. Bauer and E. Trinka, "Nonconvulsive status epilepticus and coma," Epilepsia, vol. 51, no. 2, pp. 177-190, 2010.

[12] D. Friedman, J. Claassen, and L. J. Hirsch, "Continuous electroencephalogram monitoring in the intensive care unit," Anesthesia and Analgesia, vol. 109, no. 2, pp. 506-523, 2009.

[13] N. S. Abend, D. J. Dlugos, C. D. Hahn, L. J. Hirsch, and S. T. Herman, "Use of EEG monitoring and management of nonconvulsive seizures in critically Ill patients: a survey of neurologists," Neurocritical Care, vol. 12, no. 3, pp. 382-389, 2010.

[14] M. L. Scheuer, "Continuous EEG monitoring in the intensive care unit," Epilepsia, vol. 43, no. 3, pp. 114-127, 2002.

[15] J. Claassen, L. J. Hirsch, R. G. Emerson, and S. A. Mayer, “Treatment of refractory status epilepticus with pentobarbital, propofol, or midazolam: a systematic review," Epilepsia, vol. 43, no. 2, pp. 146-153, 2002.

[16] D. H. Lowenstein, "The management of refractory status epilepticus: an update," Epilepsia, vol. 47, supplement 1, pp. 35-40, 2006.

[17] M. Holtkamp, F. Masuhr, L. Harms, K. M. Einhäupl, H. Meierkord, and K. Buchheim, "The management of refractory generalised convulsive and complex partial status epilepticus in three European countries: a survey among epileptologists and critical care neurologists," Journal of Neurology Neurosurgery and Psychiatry, vol. 74, no. 8, pp. 1095-1099, 2003.

[18] R. Kälviäinen, K. Eriksson, and I. Parviainen, "Refractory generalised convulsive status epilepticus: a guide to treatment," CNS Drugs, vol. 19, no. 9, pp. 759-768, 2005.

[19] A. R. C. Kelso and H. R. Cock, "Status epilepticus," Practical Neurology, vol. 5, pp. 322-333, 2005.

[20] H. Meierkord, P. Boon, B. Engelsen et al., "EFNS guideline on the management of status epilepticus in adults," European Journal of Neurology, vol. 17, no. 3, pp. 348-355, 2010.

[21] K. G. Jordan and L. J. Hirsch, "In nonconvulsive status epilepticus (NCSE), treat to burst-suppression: pro and Con," Epilepsia, vol. 47, no. 12, pp. 41-45, 2006.

[22] A. O. Rossetti, M. D. Reichhart, M.-D. Schaller, P.-A. Despland, and J. Bogousslavsky, "Propofol treatment of refractory status epilepticus: a study of 31 episodes," Epilepsia, vol. 45, no. 7, pp. 757-763, 2004.

[23] S. A. Mayer, J. Claassen, J. Lokin, F. Mendelsohn, L. J. Dennis, and B.-F. Fitzsimmons, "Refractory status epilepticus: frequency, risk factors, and impact on outcome," Archives of Neurology, vol. 59, no. 2, pp. 205-210, 2002.

[24] A. O. Rossetti, G. Logroscino, and E. B. Bromfield, "Refractory status epilepticus: effect of treatment aggressiveness on prognosis," Archives of Neurology, vol. 62, no. 11, pp. 1698-1702, 2005.

[25] F. W. Drislane, M. R. Lopez, A. S. Blum, and D. L. Schomer, "Detection and treatment of refractory status epilepticus in the intensive care unit," Journal of Clinical Neurophysiology, vol. 25, no. 4, pp. 181-186, 2008.

[26] N. S. Abend and D. J. Dlugos, "Treatment of refractory status epilepticus: literature review and a proposed protocol," Pediatric Neurology, vol. 38, no. 6, pp. 377-390, 2008.

[27] P. N. Varelas, "How I treat status epilepticus in the Neuro-ICU," Neurocritical Care, vol. 9, no. 1, pp. 153-157, 2008.

[28] J. Novy, G. Logroscino, and A. O. Rossetti, "Refractory status epilepticus: a prospective observational study," Epilepsia, vol. 51, no. 2, pp. 251-256, 2010.

[29] M. Tripathi, D. Vibha, N. Choudhary et al., "Management of refractory status epilepticus at a tertiary care centre in a developing country," Seizure, vol. 19, no. 2, pp. 109-111, 2010. 
[30] S. Sinha, D. K. Prashantha, K. Thennarasu, G. S. Umamaheshwara Rao, and P. Satishchandra, "Refractory status epilepticus: a developing country perspective," Journal of the Neurological Sciences, vol. 290, no. 1-2, pp. 60-65, 2010.

[31] G. B. Young, K. G. Jordan, and G. S. Doig, "An assessment of nonconvulsive seizures in the intensive care unit using continuous EEG monitoring: an investigation of variables associated with mortality," Neurology, vol. 47, no. 1, pp. 83-89, 1996.

[32] D. J. Chong and L. J. Hirsch, "Which EEG patterns warrant treatment in the critically ill? Reviewing the evidence for treatment of periodic epileptiform discharges and related patterns," Journal of Clinical Neurophysiology, vol. 22, no. 2, pp. 79-91, 2005.

[33] E. F. M. Wijdicks, "Clinical scales for comatose patients: the Glasgow Coma Scale in historical context and the new FOUR score," Reviews in Neurological Diseases, vol. 3, no. 3, pp. 109117, 2006.

[34] J. L. Banks and C. A. Marotta, "Outcomes validity and reliability of the modified rankin scale: implications for stroke clinical trials-a literature review and synthesis," Stroke, vol. 38, no. 3, pp. 1091-1096, 2007.

[35] W. A. Knaus, E. A. Draper, D. P. Wagner, and J. E. Zimmerman, "APACHE II: a severity of disease classification system," Critical Care Medicine, vol. 13, no. 10, pp. 818-829, 1985.

[36] A. O. Rossetti, G. Logroscino, T. A. Milligan, C. Michaelides, C. Ruffieux, and E. B. Bromfield, "Status Epilepticus Severity Score (STESS): a tool to orient early treatment strategy," Journal of Neurology, vol. 255, no. 10, pp. 1561-1566, 2008.

[37] N. C. Patel, I. R. Landan, J. Levin, J. Szaflarski, and A. N. Wilner, "The use of levetiracetam in refractory status epilepticus," Seizure, vol. 15, no. 3, pp. 137-141, 2006.

[38] G. Möddel, S. Bunten, C. Dobis et al., "Intravenous levetiracetam: a new treatment alternative for refractory status epilepticus," Journal of Neurology, Neurosurgery and Psychiatry, vol. 80, no. 6, pp. 689-692, 2009.

[39] S. Knake, J. Gruener, K. Hattemer et al., "Intravenous levetiracetam in the treatment of benzodiazepine refractory status epilepticus," Journal of Neurology, Neurosurgery and Psychiatry, vol. 79, no. 5, pp. 588-589, 2008.

[40] A. R. Towne, L. K. Garnett, E. J. Waterhouse, L. D. Morton, and R. J. DeLorenzo, "The use of topiramate in refractory status epilepticus," Neurology, vol. 60, no. 2, pp. 332-334, 2003.

[41] M. K. Bensalem and T. A. Fakhoury, "Topiramate and status epilepticus: report of three cases," Epilepsy and Behavior, vol. 4, no. 6, pp. 757-760, 2003.

[42] B. Soler, J. Godoy, and P. Mellado, "Treatment of refractory status epilepticus with topiramate. Report of three cases," Revista Medica de Chile, vol. 137, no. 7, pp. 936-939, 2009.

[43] M. Z. Koubeissi, S. Amina, I. Pita, G. K. Bergey, and M. A. Werz, "Tolerability and efficacy of oral loading of levetiracetam," Neurology, vol. 70, no. 22, pp. 2166-2170, 2008.

[44] F. W. Drislane, A. S. Blum, M. R. Lopez, S. Gautam, and D. L. Schomer, "Duration of refractory status epilepticus and outcome: loss of prognostic utility after several hours," Epilepsia, vol. 50, no. 6, pp. 1566-1571, 2009.

[45] A. D. Cooper, J. W. Britton, and A. A. Rabinstein, "Functional and cognitive outcome in prolonged refractory status epilepticus," Archives of Neurology, vol. 66, no. 12, pp. 1505-1509, 2009. 


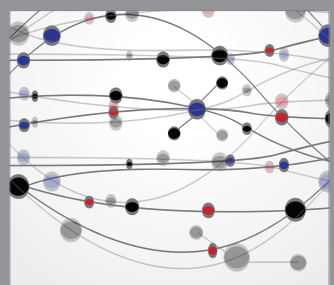

The Scientific World Journal
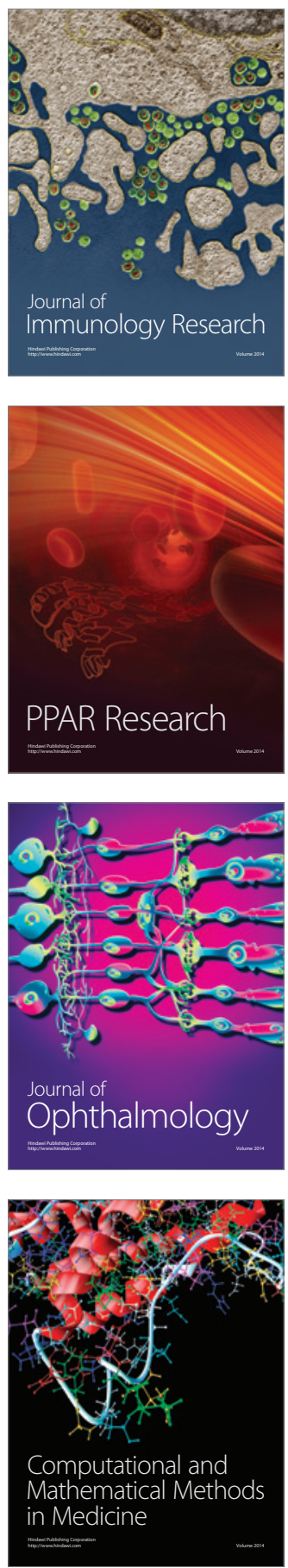

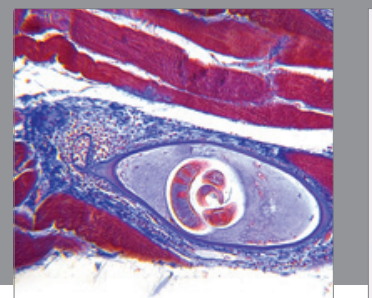

Gastroenterology

Research and Practice
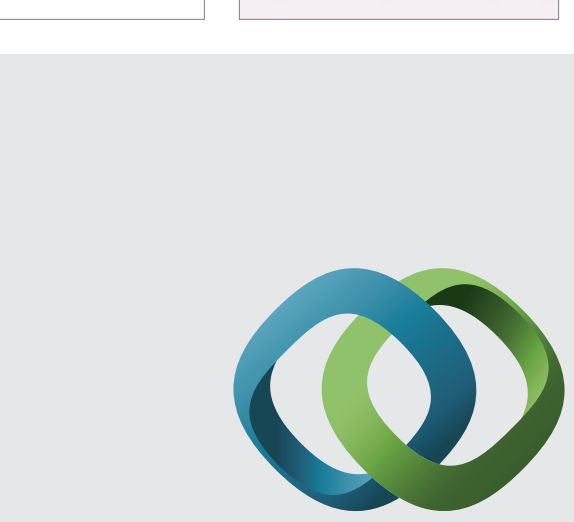

\section{Hindawi}

Submit your manuscripts at

http://www.hindawi.com
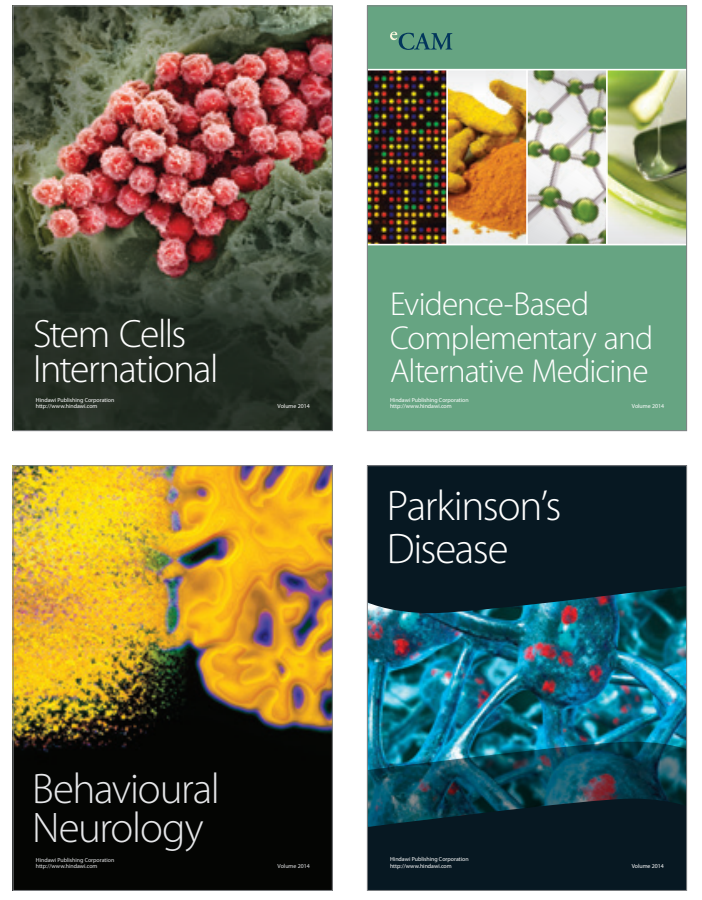
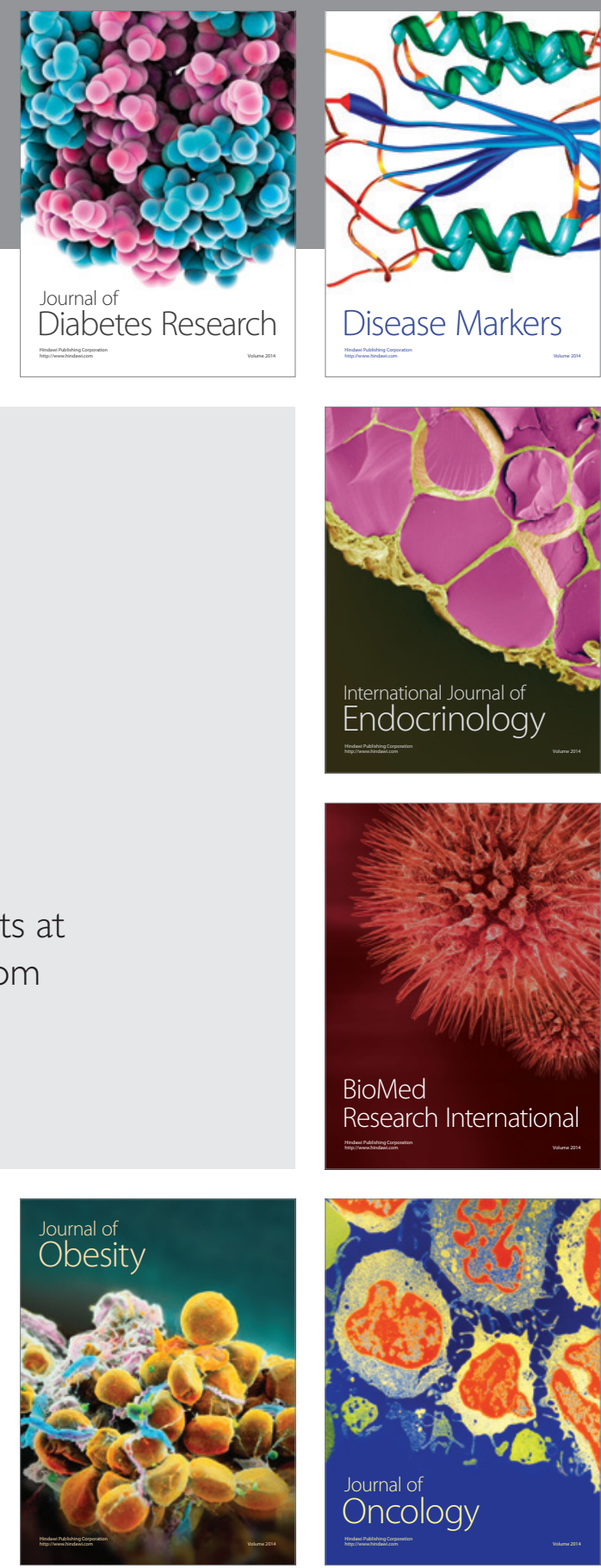

Disease Markers
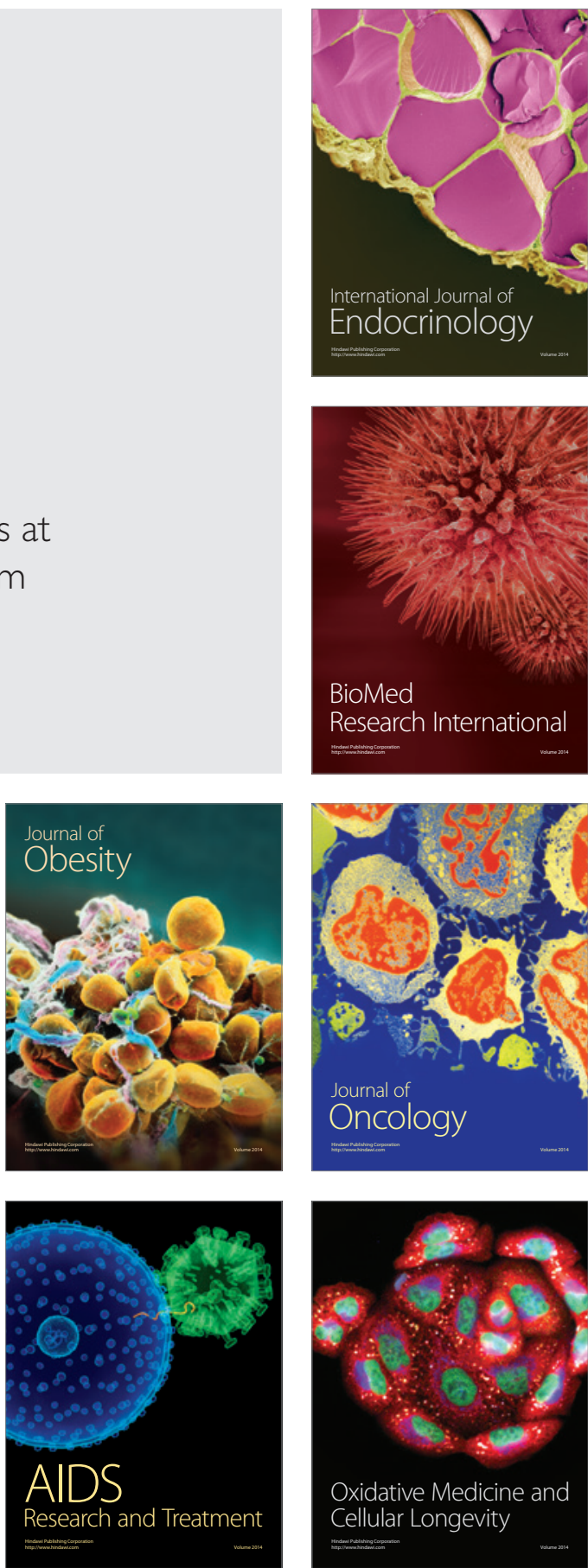